\title{
Research Article \\ Dynamic Analysis of Two Policy Lags in a Kaldorian Model
}

\author{
Eiji Tsuzuki \\ Faculty of Economics, Chiba Keizai University, Chiba 263-0021, Japan \\ Correspondence should be addressed to Eiji Tsuzuki; tsuzukie5@gmail.com
}

Received 23 May 2015; Revised 11 August 2015; Accepted 13 August 2015

Academic Editor: Mauro Sodini

Copyright (C) 2015 Eiji Tsuzuki. This is an open access article distributed under the Creative Commons Attribution License, which permits unrestricted use, distribution, and reproduction in any medium, provided the original work is properly cited.

\begin{abstract}
We examine the effects of policy lags on local economic stability using a Kaldorian model. This study analyzes two cases: the case of a monetary policy with a time lag and the case of a policy with both fiscal and monetary lags. Similar to the case of fiscal policy lags examined in a previous study, monetary policy lags have destabilizing effects on economic stability. However, in the case of the existence of both fiscal and monetary policy lags, there is a possibility that a monetary policy lag can stabilize an economy.
\end{abstract}

\section{Introduction}

In this study, we investigate the effects of policy lags (i.e., fiscal and monetary policy lags) on the stability of an economy. As the analytical framework, we employ a traditional Keynesian macrodynamic model. In general, two types of time lags have been studied: distributed and fixed lags. The present study focuses on the fixed lag (the time-to-build model developed by Kalecki [1] is the origin of economic models that consider a fixed lag; Szydłowski [2, 3] develops Kalecki's [1] model to incorporate the factors of economic growth; economic models that introduce a distributed lag include Fanti and Manfredi's [4] dynamic IS-LM model and Yoshida and Asada's [5] Keynes-Goodwin model; Yoshida and Asada also examine the case of a fixed policy lag).

One of the representative traditional Keynesian macrodynamic models is the dynamic IS-LM model developed by Schinasi [6] and Sasakura [7]. In a previous study, De Cesare and Sportelli [8] develop a dynamic IS-LM model with a tax collection lag. Furthermore, Matsumoto and Szidarovszky [9] use the dynamic IS-LM model to compare the case of a fixed lag with that of a distributed lag in tax collections and demonstrate that a larger stable region can be established in the case of a fixed lag compared with a distributed lag. Both of these studies evidently show that a policy lag restricts the ability of traditional fiscal policies to stabilize an economy.

The Kaldorian model originated in the studies of Kaldor [10] and Chang and Smyth [11] is another representative model of the traditional Keynesian macrodynamic model.
Asada and Yoshida [12] introduce a fiscal policy lag into this model and demonstrate that an increase in the responsiveness of a fiscal policy can lead to local instability, as is the case with the dynamic IS-LM model.

Recently, economic models that consider two time lags have been developed. For example, Zhou and Li [13] propose a dynamic IS-LM model with two capital accumulation lags (Zhou and Li's [13] model can be considered as an extension of Cai's [14] model, which is a dynamic IS-LM model with only one capital accumulation lag). Sportelli et al. [15] present a similar model with two time lags in the public sector: government expenditure and tax collection lags. Moreover, Matsumoto and Szidarovszky [16] propose a nonlinear multiplier-accelerator model with investment and consumption lags. These studies demonstrate that the steady state fluctuates between stability and instability with an increase in a certain policy lag; that is, multiple stability switches can occur.

In the present study, we first propose a Kaldorian model that only introduces the monetary policy lag, which can be considered as the monetary policy lag version of Asada and Yoshida's [12] model, and perform a local stability analysis. Furthermore, we propose a model that considers the existence of both fiscal and monetary policy lags. For the analysis of this model, we employ the mathematical method developed by $\mathrm{Gu}$ et al. [17]. Their method enables us to represent a stability crossing curve (a curve that separates stable and unstable regions on a lag parameter plane) as a perfectly parameterized form. Before the advent of $\mathrm{Gu}$ et al.'s 
[17] method, one could only represent one lag as a function of another lag. Using their method, we can present a more exact figure of a stability crossing curve. Few studies have employed this method for economic analysis.

This study proceeds as follows. Section 2 presents a dynamic system that represents a model economy, while Section 3 examines the local stability in the case of a monetary policy with a lag. Subsequently, Section 4 examines the case wherein both fiscal and monetary policy lags exist. Finally, Section 5 presents our conclusion.

\section{The Model}

2.1. Basic Equations. The model economy comprises the following equations:

$$
\begin{aligned}
\dot{Y}(t) & =\alpha[C(t)+I(t)+G(t)-Y(t)] ; \quad \alpha>0 \\
C(t) & =c[Y(t)-T(t)]+\bar{C} ; \quad 0<c<1 ; \bar{C}>0 \\
T(t) & =\tau Y(t)-\bar{T} ; \quad 0<\tau<1 ; \bar{T} \geq 0, \\
I(t) & =I(Y(t), K(t), r(t)) ; \\
G(t) & =\beta\left[\bar{Y}-Y\left(t-\theta_{1}\right)\right]+\bar{G} ; \\
\dot{K}(t) & =I(Y(t), K(t), r(t)), \quad \beta>0 ; \bar{Y}>0 ; \bar{G}>0 \\
\frac{M(t)}{\bar{P}} & =L(Y(t), r(t)) ; \quad \bar{P}>0 ; L_{Y}>0 ; I_{r}<0 \\
M(t) & =\bar{P} \gamma\left[\bar{Y}-Y\left(t-\theta_{2}\right)\right]+\bar{M} ; \quad \gamma>0 ; \bar{M}>0
\end{aligned}
$$

where $Y=$ real national income; $C=$ real private consumption; $I$ = real private investment; $G=$ real government expenditure; $T=$ real income tax; $K=$ real capital stock; $r$ = nominal interest rate; $M=$ nominal money supply; $\alpha=$ adjustment speed of the goods market; $c=$ marginal propensity to consume; $\bar{C}=$ base consumption; $\tau=$ marginal tax rate; $\bar{T}=$ real subsidy; $\beta=$ activeness level of fiscal policy; $\bar{Y}=$ target level of real national income; $\bar{G}=$ target level of real government expenditure; $\bar{P}=$ price level; $\gamma=$ activeness level of monetary policy; $\bar{M}=$ target level of nominal money supply; $t=$ time; $\theta_{1}=$ fiscal policy lag; and $\theta_{2}=$ monetary policy lag.

Equations (1), (2), (3), and (4) represent a disequilibrium adjustment function of the goods market, a consumption function, a tax collection function, and an investment function, respectively. Equation (5) represents a fiscal policy reaction function with a government expenditure lag, while (6) signifies a capital accumulation function. For simplicity, we assume that capital does not depreciate. Equation (7) represents the monetary market equilibrium condition, where the left-hand side denotes real money balance and the right-hand side indicates real money demand (in this study, we assume that the price level $P$ is constant; by assuming
$P(t)=P(Y(t))$ (where $P_{Y}>0$ ), we can allow for variations in the price level; however, this change does not affect the nature of our argument). Here, we ensure that the adjustment of the monetary market is rapid, and therefore the balance of demand and supply of this market is always maintained. Finally, (8) represents a monetary policy reaction function with a money supply lag.

If real money balance is constant (i.e., $\gamma=0$ ), then the system compounded from (1)-(8) essentially becomes similar to that of Asada and Yoshida [12]. However, the assumptions of $\gamma>0$ and $\theta_{2}>0$ significantly complicate the dynamic property of the system, thereby resulting in a major change in the economic implication of time lags.

2.2. Summarization. We summarize (1)-(8) in a two-dimensional dynamic system. First, by substituting (8) into (7) and solving for $r$, we obtain

$$
r(t)=r\left(Y(t), Y\left(t-\theta_{2}\right)\right),
$$

where $r_{Y(t)}=-L_{Y} / L_{r}>0$ and $r_{Y\left(t-\theta_{2}\right)}=-\gamma / L_{r}>0$.

Furthermore, by substituting (3) into (2) and substituting (9) into (4), we obtain

$$
\begin{aligned}
& C(t)=c(1-\tau) Y(t)+c \bar{T}+\bar{C}, \\
& I(t)=I\left(Y(t), K(t), r\left(Y(t), Y\left(t-\theta_{2}\right)\right)\right) .
\end{aligned}
$$

Finally, by substituting (5), (10), and (11) into (1) and substituting (11) into (6), we obtain the following system of differential equations with two time delays:

$$
\begin{aligned}
& \dot{Y}(t)=\alpha\left[I\left(Y(t), K(t), r\left(Y(t), Y\left(t-\theta_{2}\right)\right)\right)\right. \\
& -\{1-c(1-\tau)\} Y(t)-\beta Y\left(t-\theta_{1}\right)+c \bar{T}+\bar{C}+\beta \bar{Y} \\
& \quad+\bar{G}], \\
& \dot{K}(t)=I\left(Y(t), K(t), r\left(Y(t), Y\left(t-\theta_{2}\right)\right)\right) .
\end{aligned}
$$

2.3. Linearization. We assume that steady-state solutions $Y^{*}>0$ and $K^{*}>0$ that satisfy $\dot{Y}(t)=\dot{K}(t)=0$ exist and these solutions are continuous with respect to all parameters. In order to analyze the local stability around the steady-state point $\left(Y^{*}, K^{*}\right)$, we linearize the system and obtain

$$
\begin{aligned}
& \dot{\hat{Y}}(t)=\alpha\left[\left\{A_{1}-1+c(1-\tau)\right\} \widehat{Y}(t)-\beta \widehat{Y}\left(t-\theta_{1}\right)\right. \\
& \left.\quad+A_{2} \widehat{Y}\left(t-\theta_{2}\right)+I_{K} \widehat{K}(t)\right], \\
& \dot{\widehat{K}}(t)=A_{1} \widehat{Y}(t)+A_{2} \widehat{Y}\left(t-\theta_{2}\right)+I_{K} \widehat{K}(t),
\end{aligned}
$$

where $\widehat{Y}(t)=Y(t)-Y^{*}, \widehat{K}(t)=K(t)-K^{*}, A_{1}=I_{Y}+I_{r} r_{Y(t)}$, and $A_{2}=I_{r} r_{Y\left(t-\theta_{2}\right)}$.

Assuming the exponential functions $\widehat{Y}(t)=C_{1} e^{\lambda t}$ and $\widehat{K}(t)=C_{2} e^{\lambda t}$, where $C_{1}$ and $C_{2}$ are arbitrary constants and 
$\lambda$ denotes the eigenvalue, as the solutions of system (13) and substituting these into the system, we obtain

$$
\left[\begin{array}{cc}
\lambda-\alpha\left\{A_{1}-1+c(1-\tau)\right\}+\alpha \beta e^{-\theta_{1} \lambda}-\alpha A_{2} e^{-\theta_{2} \lambda} & -\alpha I_{K} \\
-A_{1}-A_{2} e^{-\theta_{2} \lambda} & \lambda-I_{K}
\end{array}\right]\left[\begin{array}{l}
\widehat{Y}(t) \\
\widehat{K}(t)
\end{array}\right]=\left[\begin{array}{l}
0 \\
0
\end{array}\right]
$$

For nontrivial solutions to exist, the determinant of the left-hand side matrix, denoted by $\Delta(\lambda)$, must be equal to zero; that is,

$$
\begin{aligned}
\Delta(\lambda)= & \lambda^{2}-\left[I_{K}+\alpha\left\{A_{1}-1+c(1-\tau)\right\}\right] \lambda \\
& -\alpha\{1-c(1-\tau)\} I_{K}+\alpha \beta\left(\lambda-I_{K}\right) e^{-\theta_{1} \lambda} \\
& -\alpha A_{2} \lambda e^{-\theta_{2} \lambda}=0 \\
= & p_{0}(\lambda)+p_{1}(\lambda) e^{-\theta_{1} \lambda}+p_{2}(\lambda) e^{-\theta_{2} \lambda}=0
\end{aligned}
$$

where

$$
\begin{aligned}
p_{0}(\lambda) & =\lambda^{2}+b_{1} \lambda+b_{2}, \\
b_{1} & =-\left[I_{K}+\alpha\left\{A_{1}-1+c(1-\tau)\right\}\right], \\
b_{2} & =-\alpha\{1-c(1-\tau)\} I_{K}>0 \\
p_{1}(\lambda) & =\alpha \beta\left(\lambda-I_{K}\right), \\
p_{2}(\lambda) & =-\alpha A_{2} \lambda
\end{aligned}
$$

Equation (15) is a characteristic equation of system (13). The significant feature of this equation is found in the existence of the exponential terms $e^{-\theta_{1} \lambda}$ and $e^{-\theta_{2} \lambda}$.

First, we examine the case of no policy lags. By substituting $\theta_{1}=\theta_{2}=0$ into (15), we obtain

$$
\lambda^{2}+\left[b_{1}+\alpha\left(\beta-A_{2}\right)\right] \lambda+b_{2}-\alpha \beta I_{K}=0,
$$

which is an ordinary quadratic equation of $\lambda$.

Thus, if $b_{1}+\alpha\left(\beta-A_{2}\right)>0$ (i.e., the coefficient of $\lambda$ from (17) is positive), the real parts of the roots of (17) are negative (see Chapter 18 in Gandolfo [18] for details regarding the relation between the roots and the coefficients of a quadric equation). In contrast, if $b_{1}+\alpha\left(\beta-A_{2}\right)<0$, the real parts of the roots are positive. Thus, if $b_{1}+\alpha\left(\beta-A_{2}\right)>0$, then the steady state is locally stable, and if $b_{1}+\alpha\left(\beta-A_{2}\right)<0$, then it is unstable.

The larger the values of $\beta$ and $\gamma$ are, the more likely the stability condition holds (note that $A_{2}=-\gamma I_{r} / L_{r}$ ). Therefore, if policy lags do not exist, then traditional fiscal and monetary policies could function effectively for stabilization.

In the following discussion, we assume that if both fiscal and monetary policy lags do not exist, then an economy is stable; that is, we have the following.

Assumption 1. We have

$$
\beta+\frac{I_{r}}{L_{r}} \gamma+\frac{b_{1}}{\alpha}>0
$$

Under this assumption, we analyze the effects of the policy lags $\left(\theta_{1}, \theta_{2}\right)$ on stability.

Incidentally, if $\left[b_{1}+\alpha\left(\beta-A_{2}\right)\right]^{2}<4\left(b_{2}-\alpha \beta I_{K}\right)$, then both roots of (17) are conjugate complex. Furthermore, by defining $\beta^{*} \equiv A_{2}-b_{1} / \alpha$, the conditions for Hopf bifurcation are satisfied at $\beta=\beta^{*}$; that is, (i) (17) includes a pair of pure imaginary roots and (ii) $d \operatorname{Re} \lambda / d \beta \neq 0$. Therefore, a cycle exists for a certain range of $\beta$ in the neighborhood $\beta^{*}$. We can develop a similar argument using the monetary policy parameter $\gamma$.

\section{Case of Monetary Policy with a Lag}

Asada and Yoshida [12] examine the situation where a time lag exists in fiscal policy responses, which is equivalent to the case wherein $\theta_{1}>0$ and $\theta_{2}=0$ in system (12). In this section, we analyze the situation where a time lag exists in monetary policy responses by assuming that $\theta_{1}=0$ and $\theta_{2}>0$ in system (12).

In this case, the characteristic equation in (15) can be rewritten as follows:

$$
\lambda^{2}+\left(b_{1}+\alpha \beta\right) \lambda+\left(b_{2}-\alpha \beta I_{K}\right)-\alpha A_{2} \lambda e^{-\theta_{2} \lambda}=0 .
$$

The following analysis is based on the study of Matsumoto and Szidarovszky [19] (Bellman and Cooke [20] provide a helpful introductory textbook of delay differential equations). The procedures of the analysis are given as follows:

(1) We characterize the points (if any) at which the dynamics around the steady state can change, that is, the points at which a zero real root or pure imaginary roots appear. These points are referred to as "crossing points."

(2) We reveal the directions of the changes in the signs of the real parts that occur when $\theta_{2}$ crosses the crossing points.

3.1. Crossing Points. First, we examine whether the points at which a zero real root appears can exist. By substituting $\lambda=0$ into (19), we obtain $b_{2}-\alpha \beta I_{K}=0$. However, since $b_{2}>0$ and $I_{K}<0$, this equality cannot hold. Accordingly, $\lambda=0$ cannot be a root.

Next, we examine whether the points at which the pure imaginary roots appear can exist. By substituting $\lambda=i \omega$, where $\omega=$ imaginary part $>0$ (pure imaginary roots are always conjugated; therefore, we can assume $\omega>0$ without loss of generality) and $i=\sqrt{-1}$, into (19), we obtain

$$
-\omega^{2}+i \omega\left(b_{1}+\alpha \beta\right)+\left(b_{2}-\alpha \beta I_{K}\right)-i \omega \alpha A_{2} e^{-i \omega \theta_{2}}=0 .
$$


Application of Euler's formula $\left(\forall x \in \mathbb{R} e^{ \pm i x}=\cos x \pm i \sin x\right)$ to this equation yields

$$
\begin{aligned}
-\omega^{2} & +\left(b_{2}-\alpha \beta I_{K}\right)-\omega \alpha A_{2} \sin \omega \theta_{2} \\
& +i\left[\left(b_{1}+\alpha \beta\right) \omega-\omega \alpha A_{2} \cos \omega \theta_{2}\right]=0 .
\end{aligned}
$$

This equality holds only when both real and imaginary parts of the left-hand side equal zero; that is,

$$
\begin{aligned}
-\omega^{2}+\left(b_{2}-\alpha \beta I_{K}\right) & =\omega \alpha A_{2} \sin \omega \theta_{2} \\
\left(b_{1}+\alpha \beta\right) \omega & =\omega \alpha A_{2} \cos \omega \theta_{2} .
\end{aligned}
$$

Or, equivalently,

$$
\begin{aligned}
& \cos \omega \theta_{2}=\frac{b_{1}+\alpha \beta}{\alpha A_{2}}, \\
& \sin \omega \theta_{2}=\frac{-\omega^{2}+b_{2}-\alpha \beta I_{K}}{\alpha A_{2} \omega} .
\end{aligned}
$$

For values of $\omega \theta_{2}$ that satisfy these equations, (19) includes pure imaginary roots, where $2 \pi h<\omega \theta_{2}<2 \pi(1+h), h=$ $0,1,2,3, \ldots$.

Furthermore, the sum of the squares of (22) yields the following:

$$
\begin{aligned}
\omega^{4}- & \left\{2\left(b_{2}-\alpha \beta I_{K}\right)-\left(b_{1}+\alpha \beta\right)^{2}+\left(\alpha A_{2}\right)^{2}\right\} \omega^{2} \\
+ & \left(b_{2}-\alpha \beta I_{K}\right)^{2}=0 .
\end{aligned}
$$

Solving for $\omega^{2}$, we obtain

$$
\omega_{ \pm}^{2}=\frac{2\left(b_{2}-\alpha \beta I_{K}\right)-\left(b_{1}+\alpha \beta\right)^{2}+\left(\alpha A_{2}\right)^{2} \pm \sqrt{N}}{2}
$$

where $N \equiv\left\{2\left(b_{2}-\alpha \beta I_{K}\right)-\left(b_{1}+\alpha \beta\right)^{2}+\left(\alpha A_{2}\right)^{2}\right\}^{2}-4\left(b_{2}-\alpha \beta I_{K}\right)^{2}$.

We denote real and positive values of $\omega$ by $\omega^{*}$. By substituting $\omega^{*}$ into (23) and solving for $\theta_{2}$, we obtain

$$
\begin{aligned}
\theta_{2 h}=\frac{\cos ^{-1}\left(\left(b_{1}+\alpha \beta\right) / \alpha A_{2}\right)+2 \pi h}{\omega^{*}} & \\
& h=0,1,2,3, \ldots .
\end{aligned}
$$

Thus, there exists an infinite number of values of $\theta_{2}$ (i.e., $\left.\theta_{20}, \theta_{21}, \theta_{22}, \ldots\right)$ that generate pure imaginary roots (i.e., crossing points).

Finally, if a real and positive value of $\omega$ does not exist, then changes in the signs of the real parts of the roots do not occur. Therefore, in this case, a policy lag does not affect stability.

Direction of Crossing. Here, we reveal how the signs of the complex roots change when $\theta_{2} \operatorname{crosses} \theta_{2 h}$. This is determined by the sign of $d \operatorname{Re} \lambda /\left.d \theta_{2}\right|_{\lambda=i \omega^{*}}$. If $d \operatorname{Re} \lambda /\left.d \theta_{2}\right|_{\lambda=i \omega^{*}}>0$, then the signs of the real parts of the roots change from negative to positive with an increase in $\theta_{2}$ (which indicates destabilization). In contrast, if $d \operatorname{Re} \lambda /\left.d \theta_{2}\right|_{\lambda=i \omega^{*}}<0$, then the signs of the real parts of the roots change from positive to negative with an increase in $\theta_{2}$ (which indicates stabilization).
TABLE 1: Baseline parameters.

\begin{tabular}{ccccc}
\hline$\alpha$ & $c$ & $\tau$ & $\bar{C}+c \bar{T}+\bar{G}$ & $\bar{Y}$ \\
\hline 0.9 & 0.625 & 0.2 & 200 & 400 \\
\hline
\end{tabular}

For convenience of calculation, we will observe the sign of $\left.\operatorname{Re}\left(d \lambda / d \theta_{2}\right)^{-1}\right|_{\lambda=i \omega^{*}}$ instead of that of $d \operatorname{Re} \lambda /\left.d \theta_{2}\right|_{\lambda=i \omega^{*}}$.

Using (19), we can demonstrate that $\left.\operatorname{Re}\left(d \lambda / d \theta_{2}\right)^{-1}\right|_{\lambda=i \omega^{*}}>$ 0 holds for any value of $h$ (see the appendix). Thus, with any crossing, the signs of the real parts of the roots change from negative to positive.

If a policy lag does not present $\left(\theta_{2}=0\right)$, then roots with positive real parts do not exist under Assumption 1. With increases in $\theta_{2}$, the number of roots with positive real parts increases by two. Accordingly, we can state that $2 h$ roots with positive real parts exist in the region of $\theta_{2} \in\left(\theta_{2(h-1)}, \theta_{2 h}\right)$ (where $\theta_{2(-1)}=0$ ). Therefore, we can offer the following proposition.

Proposition 2. For $\theta_{2} \in\left(0, \theta_{20}\right)$, the steady state is stable, and, for $\theta_{2} \in\left(\theta_{20}, \infty\right)$, it is unstable.

This proposition suggests that even if a monetary policy is sufficiently active enough to satisfy Assumption 1, an economy can become unstable due to the existence of a policy lag. A similar result has already been shown by Asada and Yoshida [12] concerning fiscal policies.

3.2. Numerical Simulations. Let us consider a numerical example. Following the study of Asada and Yoshida [12], we set baseline parameters, as shown in Table 1 .

Furthermore, Asada and Yoshida [12] employ the investment function that is highly nonlinear with respect to $Y(t)$, which is formulated as follows (in their model, $\gamma=0$ ):

$$
\begin{array}{r}
I=\frac{\bar{Y}}{1+12 e^{-0.1(Y(t)-\bar{Y})}}-r-0.5 K(t)-\frac{\bar{Y}}{13}+0.2+30, \\
r=-0.05 \gamma\left(\bar{Y}-Y\left(t-\theta_{2}\right)\right)+0.01 \sqrt{Y(t)} .
\end{array}
$$

Based on these assumptions, the steady-state values are provided by $\left(Y^{*}, K^{*}\right)=(400,60)$.

We also set the baseline values of $\beta$ and $\gamma$ as 2.5 and 6.0 , respectively (generally, it seems that the responsiveness of monetary policies to economic fluctuations is often larger than that of fiscal policies; therefore, we assume $\gamma>\beta$ ). When $\gamma$ is fixed at the baseline value, $\omega^{*}$ exists only for $\beta \epsilon$ $(1.49,2.08)$. Outside this range, a policy lag does not affect stability. In addition, Assumption 1 is satisfied for $\beta>1.49$. Accordingly, irrespective of the value of a monetary policy lag, the steady state is unstable for $0<\beta<1.49$, and it is stable for $\beta>2.08$.

Likewise, when $\beta$ is fixed at the baseline value, $\omega^{*}$ exists for $\gamma>14.32$. In addition, Assumption 1 is satisfied for $\gamma>$ -14.32 . Hence, the steady state is stable for $0<\gamma<14.32$, irrespective of the value of a lag.

Figure 1 describes $\theta_{2 h}(h=0,1,2)$ that corresponds to $\omega^{*}$ as a function of $\beta$ and $\gamma$. These curves are referred to as 

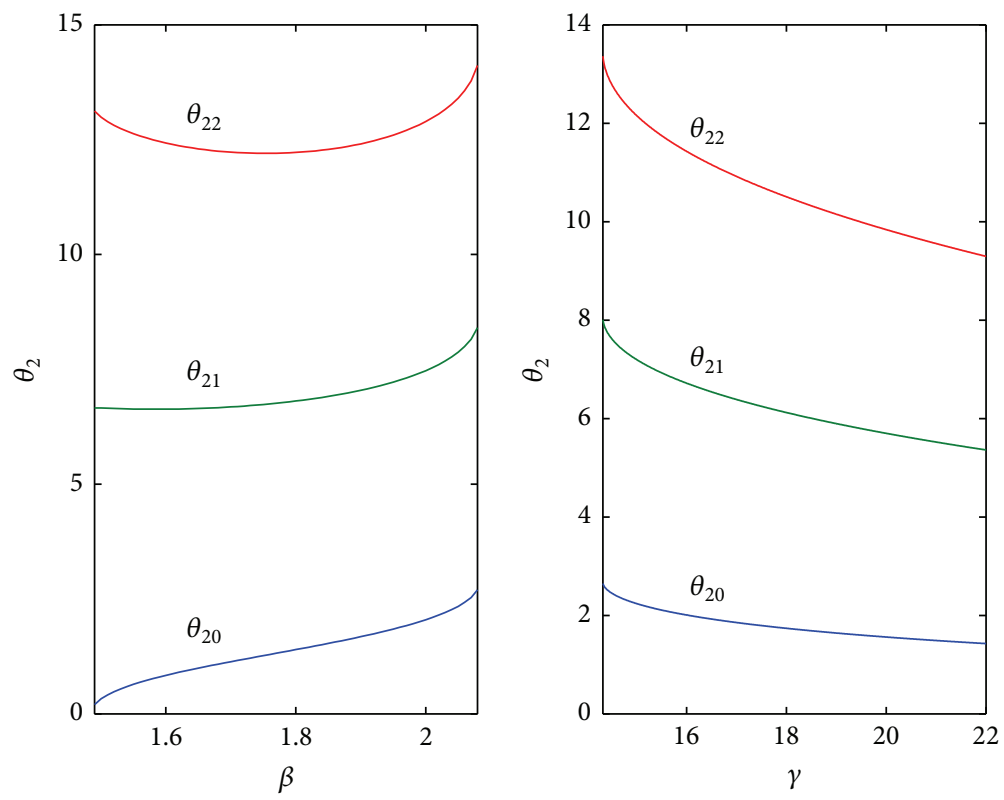

Figure 1: Crossing curves.

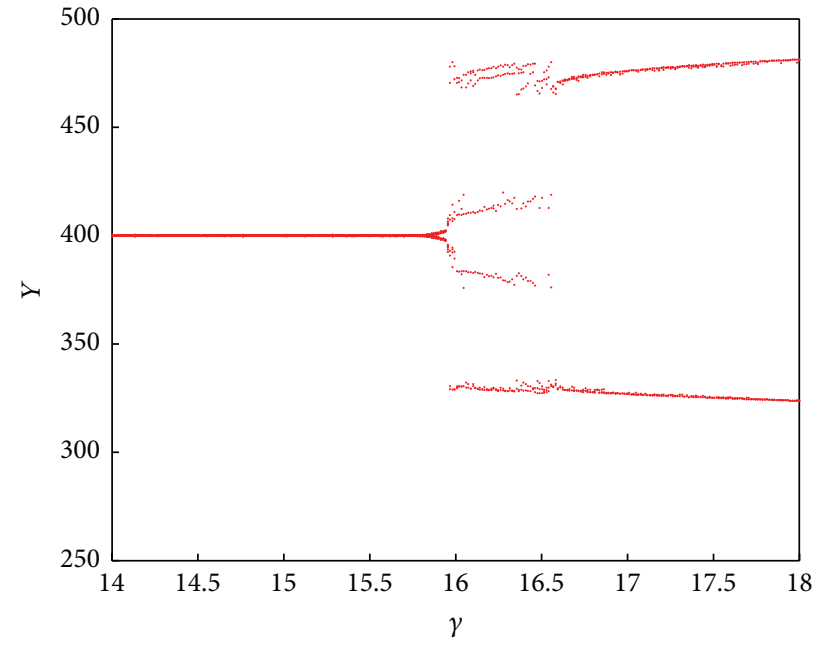

FIGURE 2: Bifurcation diagram (case with (28)).

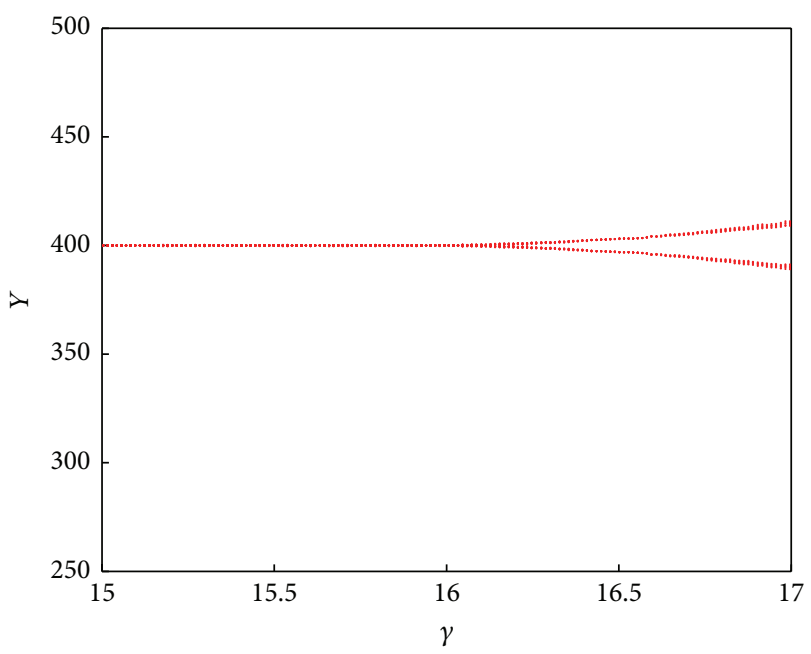

FIGURE 3: Bifurcation diagram (case with (29)).

and minimal values of $Y(t)$ ). This figure shows that not only one period but also multiple period cycles can emerge. The emergence of multiple period cycles is attributed to the strong nonlinearity of the investment function with respect to $Y(t)$. When we use the investment function that has relatively simpler form with respect to $Y(t)$ as below (we set the coefficient of $\sqrt{Y(t)}$ as 113.6 so that the value of $I_{Y}$ equals that of (28)), the bifurcation diagram becomes as shown in Figure 3. In this case, only one period cycle exists. Hence,

$$
\begin{aligned}
I= & 113.6 \sqrt{Y(t)}-r-0.5 K(t)-113.6 \sqrt{\bar{Y}}+0.2 \\
& +30 .
\end{aligned}
$$
a bifurcation diagram can be described for $\gamma \in[14,18]$, as shown in Figure 2 (the vertical axis represents the maximal 
Although Asada and Yoshida [12] do not clearly specify the time dimension, similar models that include a disequilibrium adjustment function of the goods market ( $\alpha$ is set around unity) specify the measure of $t$ as a year (refer to, e.g., Yoshida and Asada [5]). Therefore, we will consider the time dimension as a year. For example, when $\beta=1.8$, the steady state is unstable for $\theta_{2}>1.4$ years.

\section{Case of Policies with Fiscal and Monetary Policy Lags}

In this section, we investigate the case wherein both fiscal and monetary policy lags exist (i.e., $\theta_{1}>0$ and $\theta_{2}>0$ ). In order to examine the signs of the roots of (15), we use a mathematical method developed by Gu et al. [17].

4.1. Preconditions. First, to apply the technique of $\mathrm{Gu}$ et al. [17], some preconditions should be examined. According to their study, (15) should satisfy the following conditions:

(I) $\operatorname{deg}\left(p_{0}(\lambda)\right) \geq \max \left\{\operatorname{deg}\left(p_{1}(\lambda)\right), \operatorname{deg}\left(p_{2}(\lambda)\right)\right\}$.

(II) $\Delta(0) \neq 0$.

(III) A solution common to all three polynomials $p_{0}(\lambda)=$ $0, p_{1}(\lambda)=0$, and $p_{2}(\lambda)=0$ does not exist.

(IV) $\lim _{\lambda \rightarrow \infty}\left(\left|p_{1}(\lambda) / p_{0}(\lambda)\right|+\left|p_{2}(\lambda) / p_{0}(\lambda)\right|\right)<1$.

Condition (I) is satisfied by $2>\max \{1,1\}$, while Condition (II) is also satisfied by $\Delta(0)=-\alpha I_{K}\{1-c(1-\tau)+\beta\}>0$. Condition (III) is obviously satisfied. Finally, Condition (IV) is satisfied by $\lim _{\lambda \rightarrow \infty}\left(\left|p_{1}(\lambda) / p_{0}(\lambda)\right|+\left|p_{2}(\lambda) / p_{0}(\lambda)\right|\right)=0$.

Now, we examine the effects of lags $\left(\theta_{1}, \theta_{2}\right)$ on the stability of the steady state. The analysis proceeds as follows:

(1) We characterize the sets $\left(\theta_{1}, \theta_{2}\right)$ (i.e., crossing sets) at which the pure imaginary roots appear (it is ensured from precondition (II) that zero cannot be a root).

(2) We depict the crossing sets on the $\left(\theta_{1}, \theta_{2}\right)$ plane (which we refer to as the crossing curves) via numerical simulation.

(3) We reveal the directions of changes in the signs of the real parts that occur when lags $\left(\theta_{1}, \theta_{2}\right)$ cross the crossing curves.

4.2. Crossing Sets. Dividing (15) by $p_{0}(\lambda)$, we obtain

$$
1+a_{1}(\lambda) e^{-\theta_{1} \lambda}+a_{2}(\lambda) e^{-\theta_{2} \lambda}=0
$$

where

$$
\begin{aligned}
& a_{1}(\lambda)=\frac{p_{1}(\lambda)}{p_{0}(\lambda)}=\frac{\alpha \beta\left(\lambda-I_{K}\right)}{\lambda^{2}+b_{1} \lambda+b_{2}}, \\
& a_{2}(\lambda)=\frac{p_{2}(\lambda)}{p_{0}(\lambda)}=-\frac{\alpha A_{2} \lambda}{\lambda^{2}+b_{1} \lambda+b_{2}} .
\end{aligned}
$$

Denoting a pure imaginary root by $\lambda=i \omega$, the values of $\omega$ that satisfy (30) can be characterized by the following lemma.

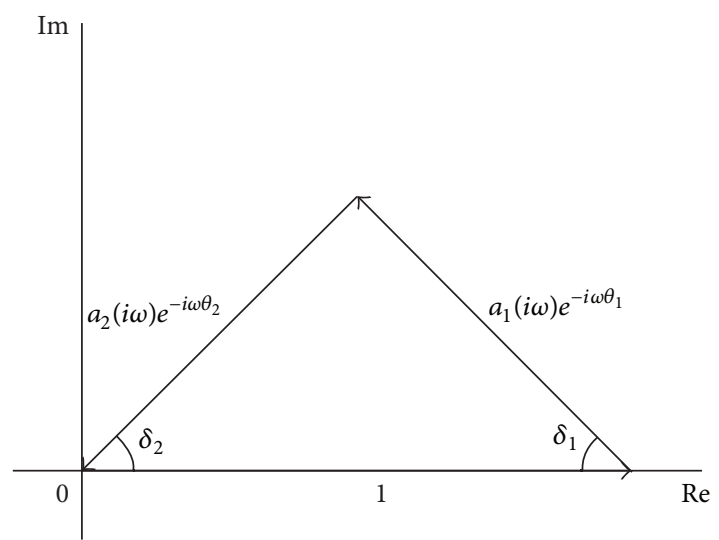

FIGURE 4: Triangle formed by $1,\left|a_{1}(i \omega)\right|$, and $\left|a_{2}(i \omega)\right|$ on the complex plane.

Lemma 3 (see [17, Proposition 3.1]). For each $\omega$ satisfying $p_{0}(i \omega) \neq 0, \lambda=i \omega$ is a solution of $\Delta(\lambda)=0$ for some $\left(\theta_{1}, \theta_{2}\right) \in \mathbb{R}_{+}^{2}$ if and only if

$$
\begin{aligned}
\left|a_{1}(i \omega)\right|+\left|a_{2}(i \omega)\right| & \geq 1, \\
-1 & \leq\left|a_{1}(i \omega)\right|-\left|a_{2}(i \omega)\right| \leq 1 .
\end{aligned}
$$

We denote the set of $\omega>0$ that satisfy conditions (32) as $\Omega$, which is termed the "crossing frequency." For all $\omega \in$ $\Omega$, the sets $\left(\theta_{1}, \theta_{2}\right)$ satisfying (30) (i.e., crossing sets) should satisfy the following conditions (Figure 4 ):

$$
\begin{aligned}
& \mp \delta_{1}=\arg \left(a_{1}(i \omega) e^{-i \omega \theta_{1}}\right)+2 m \pi ; \quad m=0,1,2, \ldots, \\
& \pm \delta_{2}=\arg \left(a_{2}(i \omega) e^{-i \omega \theta_{2}}\right)+2 n \pi ; \quad n=0,1,2, \ldots,
\end{aligned}
$$

where $\delta_{1}, \delta_{2} \in[0, \pi]$.

Incidentally, on the complex plane, multiplication of amplitudes becomes a sum of the parts; therefore, we obtain

$$
\begin{aligned}
& \arg \left(a_{1}(i \omega) e^{-i \omega \theta_{1}}\right)=\arg \left(a_{1}(i \omega)\right)-\omega \theta_{1}, \\
& \arg \left(a_{2}(i \omega) e^{-i \omega \theta_{2}}\right)=\arg \left(a_{2}(i \omega)\right)-\omega \theta_{2} .
\end{aligned}
$$

In addition, Figure 4 demonstrates that the following relations hold:

$$
\begin{aligned}
& \arg \left(a_{1}(i \omega)\right)=\tan ^{-1}\left(\frac{\operatorname{Im}\left(a_{1}(i \omega)\right)}{\operatorname{Re}\left(a_{1}(i \omega)\right)}\right), \\
& \arg \left(a_{2}(i \omega)\right)=\tan ^{-1}\left(\frac{\operatorname{Im}\left(a_{2}(i \omega)\right)}{\operatorname{Re}\left(a_{2}(i \omega)\right)}\right) .
\end{aligned}
$$

Moreover, after some manipulation, (31) derive the following expressions:

$$
\begin{aligned}
& \frac{\operatorname{Im}\left(a_{1}(i \omega)\right)}{\operatorname{Re}\left(a_{1}(i \omega)\right)}=\frac{b_{1} \omega I_{K}+\omega\left(b_{2}-\omega^{2}\right)}{b_{1} \omega^{2}-I_{K}\left(b_{2}-\omega^{2}\right)}, \\
& \frac{\operatorname{Im}\left(a_{2}(i \omega)\right)}{\operatorname{Re}\left(a_{2}(i \omega)\right)}=\frac{b_{2}-\omega^{2}}{b_{1} \omega} .
\end{aligned}
$$


Thus, using (34) to (36), (33) can be rewritten as follows:

$$
\begin{aligned}
& \theta_{1}=\frac{\tan ^{-1}\left(\left(b_{1} \omega I_{K}+\omega\left(b_{2}-\omega^{2}\right)\right) /\left(b_{1} \omega^{2}-I_{K}\left(b_{2}-\omega^{2}\right)\right)\right) \pm \delta_{1}+2 m \pi}{\omega}, \\
& \theta_{2}=\frac{\tan ^{-1}\left(\left(b_{2}-\omega^{2}\right) / b_{1} \omega\right) \mp \delta_{2}+2 n \pi}{\omega},
\end{aligned}
$$

where the interior angles of the triangle denoted by $\delta_{1}$ and $\delta_{2}$ are given by the cosine theorem as follows:

$$
\begin{aligned}
& \delta_{1}=\cos ^{-1}\left(\frac{1+\left|a_{1}(i \omega)\right|^{2}-\left|a_{2}(i \omega)\right|^{2}}{2\left|a_{1}(i \omega)\right|}\right)=\cos ^{-1}\left(\frac{\left(b_{2}-\omega^{2}\right)^{2}+\left(b_{1} \omega\right)^{2}+\left(\alpha \beta I_{K}\right)^{2}+(\alpha \beta \omega)^{2}-\left(\alpha A_{2} \omega\right)^{2}}{2 \sqrt{\left(\alpha \beta I_{K}\right)^{2}+(\alpha \beta \omega)^{2}} \sqrt{\left(b_{2}-\omega^{2}\right)^{2}+\left(b_{1} \omega\right)^{2}}}\right), \\
& \delta_{2}=\cos ^{-1}\left(\frac{1+\left|a_{2}(i \omega)\right|^{2}-\left|a_{1}(i \omega)\right|^{2}}{2\left|a_{2}(i \omega)\right|}\right)=\cos ^{-1}\left(\frac{\left(b_{2}-\omega^{2}\right)^{2}+\left(b_{1} \omega\right)^{2}+\left(\alpha A_{2} \omega\right)^{2}-\left(\alpha \beta I_{K}\right)^{2}-(\alpha \beta \omega)^{2}}{2 \sqrt{\left(\alpha A_{2} \omega\right)^{2}} \sqrt{\left(b_{2}-\omega^{2}\right)^{2}+\left(b_{1} \omega\right)^{2}}}\right) .
\end{aligned}
$$

Equations (37) characterize the crossing sets $\left(\theta_{1}, \theta_{2}\right) \in$ $\mathbb{R}_{+}^{2}$. Depending on the signs of $\delta_{1}$ and $\delta_{2}$, we can define two types of crossing sets, denoted by $L_{1}(m, n)$ and $L_{2}(m, n)$, as follows:

$$
\begin{aligned}
& \theta_{1}=\frac{\tan ^{-1}\left(\left(b_{1} \omega I_{K}+\omega\left(b_{2}-\omega^{2}\right)\right) /\left(b_{1} \omega^{2}-I_{K}\left(b_{2}-\omega^{2}\right)\right)\right)+\delta_{1}+2 m \pi}{\omega}, \\
& \theta_{2}=\frac{\tan ^{-1}\left(\left(b_{2}-\omega^{2}\right) / b_{1} \omega\right)-\delta_{2}+2 n \pi}{\omega}, \\
& \theta_{1}=\frac{\tan ^{-1}\left(\left(b_{1} \omega I_{K}+v\left(b_{2}-\omega^{2}\right)\right) /\left(b_{1} \omega^{2}-I_{K}\left(b_{2}-\omega^{2}\right)\right)\right)-\delta_{1}+2 m \pi}{\omega}, \\
& \theta_{2}=\frac{\tan ^{-1}\left(\left(b_{2}-\omega^{2}\right) / b_{1} \omega\right)+\delta_{2}+2 n \pi}{\omega} .
\end{aligned}
$$

As in the previous section, we set parameter values and the functional form of the investment function, as shown in Table 1, (28) or (29), $\beta=2.5$ and $\gamma=6.0$. In this case, crossing frequency $\Omega$ is provided by $\omega \in(1.50,2.13)$ (Figure 5) (according to Gu et al.s [17] classification, Figure 5 exhibits Type 13). For $\omega \in \Omega$, describing the crossing sets $L_{1}(m, n)$ and $L_{2}(m, n)$ on the $\theta_{1}-\theta_{2}$ plane, we obtain Figure 6, where $m=0,1,2$ and $n=0,1,2$. The dotted curves represent $L_{1}(m, n)$, and the solid curves represent $L_{2}(m, n)$. The points marked by a dot indicate the starting points of both curves, that is, the points corresponding to $\omega=1.50$.
We establish how the real parts of the roots change when lags cross the crossing curves with an increase in $\theta_{1}$. For $\omega \in$ $\Omega$, if $\operatorname{Re}\left(d \lambda / d \theta_{1}\right)^{-1}>0$, then the real parts of the roots change from negative to positive; this indicates destabilization. In contrast, if $\operatorname{Re}\left(d \lambda / d \theta_{1}\right)^{-1}<0$, then the real parts of the roots change from positive to negative; this indicates stabilization.

Differentiating (30) with respect to $\theta_{1}$, we obtain

$$
\begin{gathered}
{\left[a_{1}^{\prime}(\lambda) e^{-\theta_{1} \lambda}-a_{1}(\lambda) e^{-\theta_{1} \lambda} \theta_{1}+a_{2}^{\prime}(\lambda) e^{-\theta_{2} \lambda}\right.} \\
\left.-a_{2}(\lambda) e^{-\theta_{2}} \theta_{2}\right] \frac{d \lambda}{d \theta_{1}}=a_{1}(\lambda) e^{-\theta_{1} \lambda} \lambda
\end{gathered}
$$




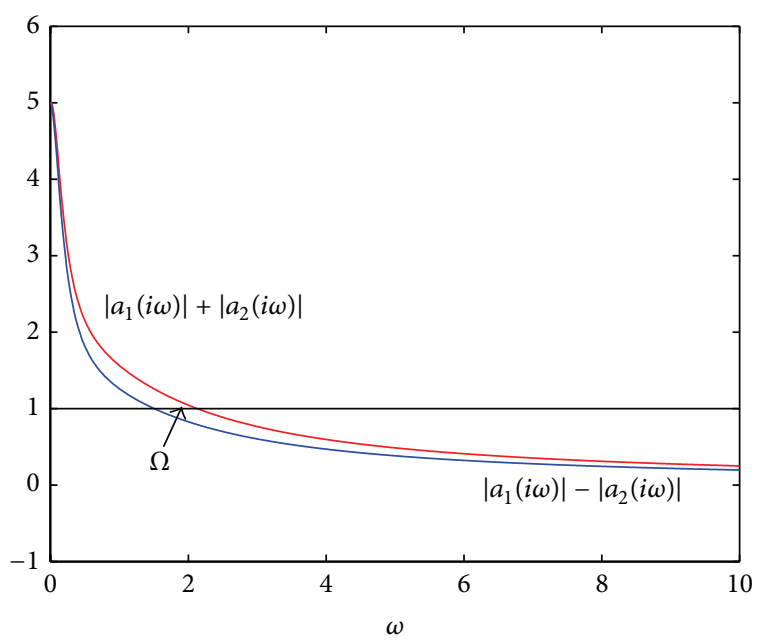

FIGURE 5: Crossing frequency $\Omega$.

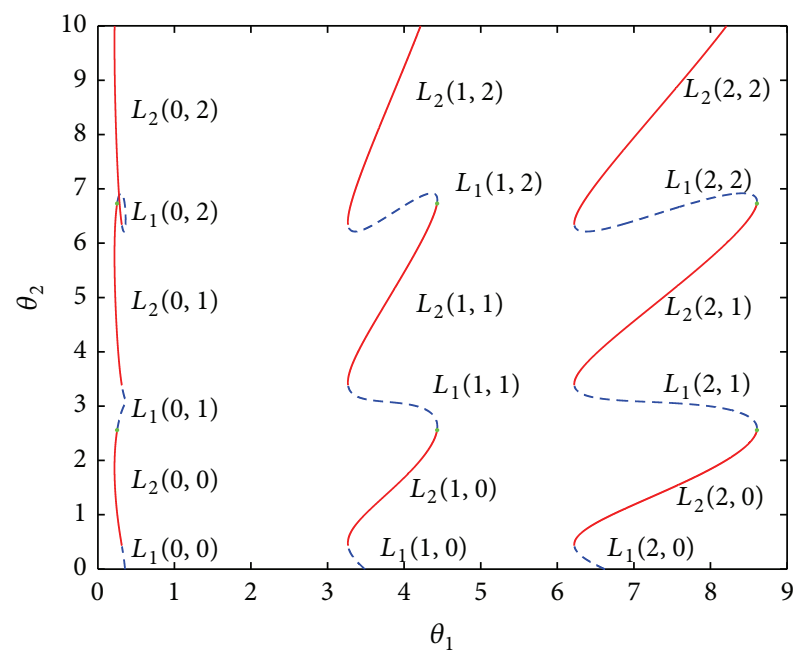

Figure 6: Crossing curves.

or, equivalently,

$$
\begin{aligned}
& \left(\frac{d \lambda}{d \theta_{1}}\right)^{-1} \\
& \quad=\frac{a_{1}^{\prime}(\lambda) e^{-\theta_{1} \lambda}+a_{2}^{\prime}(\lambda) e^{-\theta_{2} \lambda}-a_{2}(\lambda) e^{-\theta_{2} \lambda} \theta_{2}}{a_{1}(\lambda) e^{-\theta_{1} \lambda} \lambda}-\frac{\theta_{1}}{\lambda},
\end{aligned}
$$

where

$$
\begin{aligned}
& a_{1}^{\prime}(\lambda)=\frac{\alpha \beta p_{0}(\lambda)-\alpha \beta\left(\lambda-I_{K}\right)\left(2 \lambda+b_{1}\right)}{p_{0}(\lambda)^{2}}, \\
& a_{2}^{\prime}(\lambda)=-\frac{\alpha A_{2} p_{0}(\lambda)-\alpha A_{2} \lambda\left(2 \lambda+b_{1}\right)}{p_{0}(\lambda)^{2}} .
\end{aligned}
$$

Describing the real parts of (40) as a function of $\omega \in \Omega$, we obtain Figure 7, where the dotted curves are the functions evaluated on curve $L_{1}$ and the solid curves are the functions evaluated on curve $L_{2}$. As for curve $L_{1}$, stabilization only occurs on the intermediate region of the segments $L_{1}(m, 2)$; destabilization occurs on the other segments of $L_{1}$. As for curve $L_{2}$, destabilization occurs on all segments. Here, we define a curve formed by connecting curves $L_{j}(0, n)$ (where $j=1,2$ and $n=0,1,2$ ) as $m 0$ (Figure 8). We also define a curve formed by connecting the points on curve $m 0$ that minimize the value of $\theta_{1}$ for a given $\theta_{2}$ as curve $M 0$. Then, we can express the stability condition as follows: set $\left(\theta_{1}, \theta_{2}\right)$ exists in the left-hand side region of curve $M 0$.

On curve $M 0$, conditions for Hopf bifurcation are satisfied, and therefore a cycle emerges. In addition, as is obvious from Figure 6, an increase in the fiscal policy lag $\theta_{1}$ necessarily destabilizes the steady state. However, an increase in the monetary policy lag $\theta_{2}$ has the potential to stabilize the steady state, which depends on the value of $\theta_{1}$. When $\theta_{1} \in(0.22,0.36)$, we can see that the steady state fluctuates between stability and instability with an increase in $\theta_{2}$. Such a phenomenon cannot be observed in models with only one time lag. Moreover, Figure 9 presents a bifurcation diagram

wherein $\theta_{1}=0.25$ and (28) is used as the investment function. This figure evidently indicates that alternations of stability and instability emerge with an increase in $\theta_{2}$.

In particular, when $\theta_{1}=0.25$, stability can be established by setting $\theta_{2} \in(0,1.06), \theta_{2} \in(2.53,4.33)$, or $\theta_{2} \in(6.71,7.61)$; that is, for equilibrium stability, monetary policy lag should be less than one year, between 3 and 4.5 years, or between 6.8 and 7.5 years when a fiscal policy lag is approximately three months.

Next, we examine how $\beta$ and $\gamma$ affect the stability condition. Both $\beta$ and $\gamma$ affect $L_{1}$ and $L_{2}$ through $\omega \in \Omega$. It is difficult to analytically show how $L_{1}$ and $L_{2}$ change. Hence, we numerically examine how curve $m 0$ (which comprises $L_{1}(0, n)$ and $\left.L_{2}(0, n)\right)$ shifts when $\beta$ and $\gamma$ increase from each baseline value $(\beta=2.5$ and $\gamma=6.0)$.

Figures 10 and 11 describe curve $m 0$ in cases with $\beta=8$ and $\beta=15$, respectively. We can observe that an increase in $\beta$ shifts curve $m 0$ to left. Accordingly, the stability region $S$ reduces. Likewise, Figures 12 and 13 describe curve $m 0$ in cases with $\gamma=12$ and $\gamma=22$, respectively. The stability region is also reduced by an increase in $\gamma$. These results suggest that increases in the activeness levels of fiscal and monetary policies increase economic instability, as is the case with only one policy lag.

4.3. Investment and Stability. In general, the formulation of the investment function has great significance to stability in macrodynamic models; that is, the larger the marginal propensity to investment is, the more likely it becomes unstable. In this subsection, we examine the effects of changes in the partial derivatives of the investment function, $I_{Y}$ and $I_{K}$, on stability. We compare three cases: the case of no policy lags, the case of the existence of only monetary policy lag, and the case of the existence of both fiscal and monetary policy lags.

In the case with no policy lags, $I_{Y}$ and $I_{K}$ affect the stability condition given by Assumption 1 through $b_{1}$. The smaller the values of $I_{Y}$ and $I_{K}$ are (the larger the absolute value of $I_{K}$ is), 

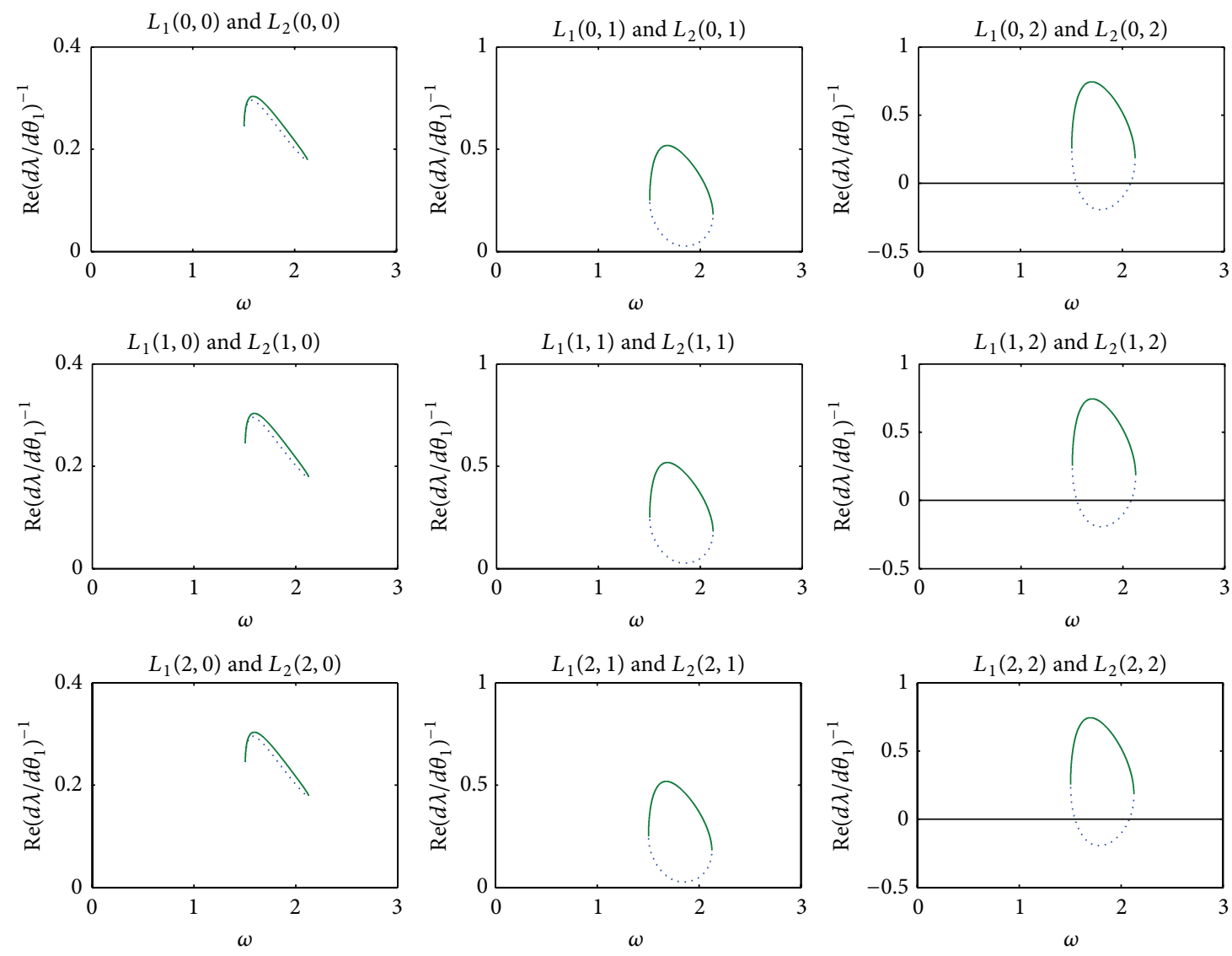

FIGURE 7: Direction of crossing.

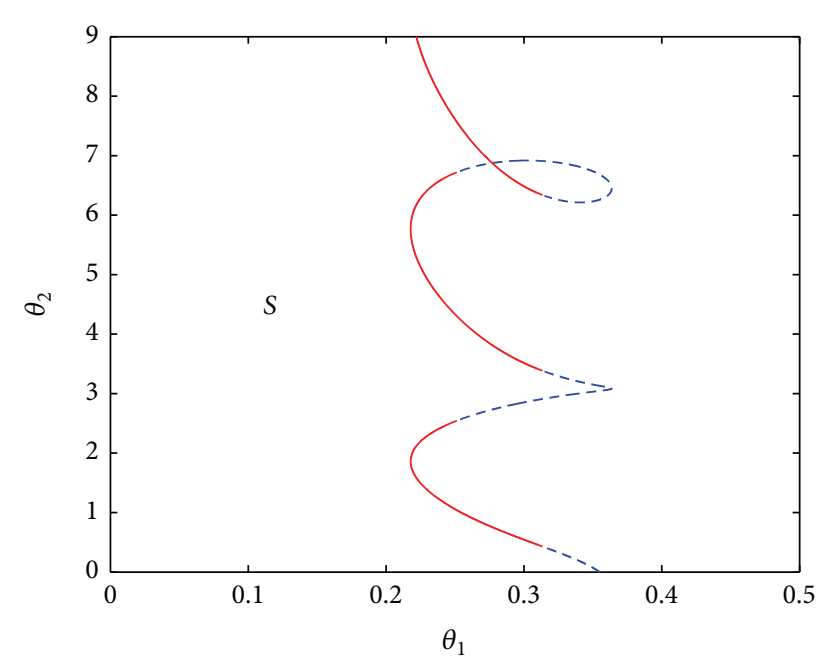

FIgURE 8: Stability region $S$.

the more likely the stability condition is satisfied. These are fairly typical results.

In the case of a monetary policy with a lag, $I_{Y}$ and $I_{K}$ affect the value of $\omega^{*}$ through $b_{2}\left(I_{K}\right.$ also directly affects $\left.\omega^{*}\right)$. Accordingly, the value of (27) changes. In this case, it is difficult to analytically show the quality and quantity of

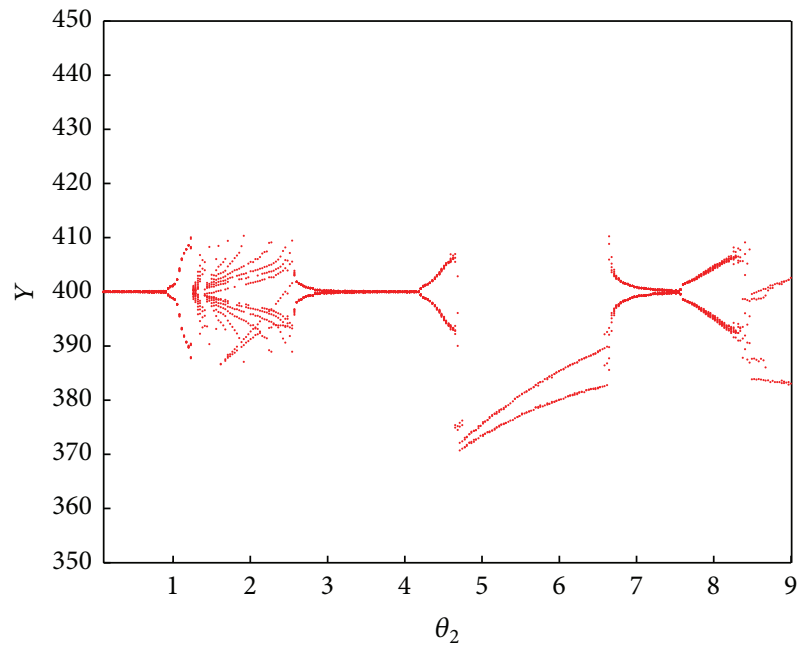

FIGURE 9: Bifurcation diagram $\left(\theta_{1}=0.25\right)$.

these effects. Hence, we resort to a numerical method. Based on the parameter values proposed in Section 3.2, crossing curves $\theta_{20}$ can be described within each range of $I_{Y}$ and $I_{K}$ that ensure the existence of $\omega^{*}$, as shown in Figure 14. The region located below these curves indicates the stability regions. Similar to the case with no policy lags, an increase 


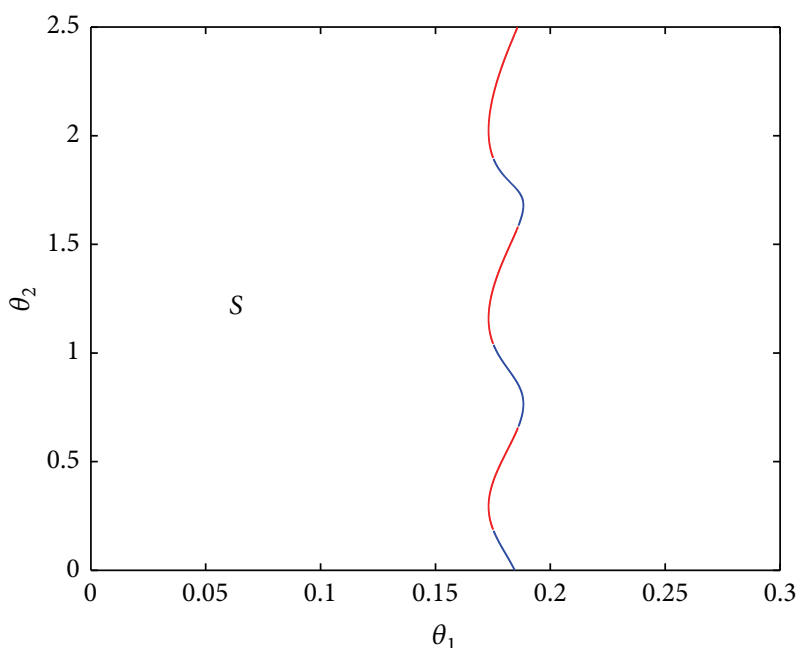

Figure 10: $\beta=8$.

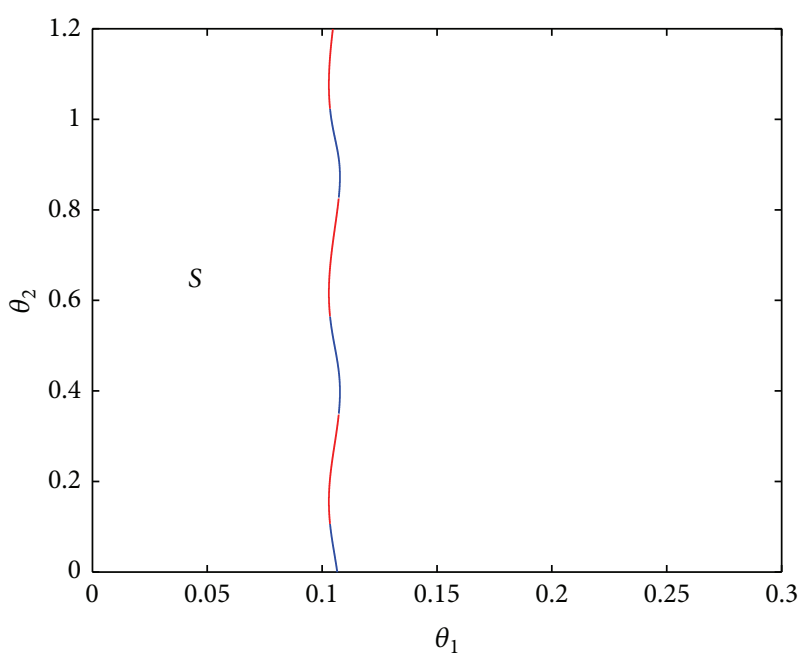

Figure 11: $\beta=15$.

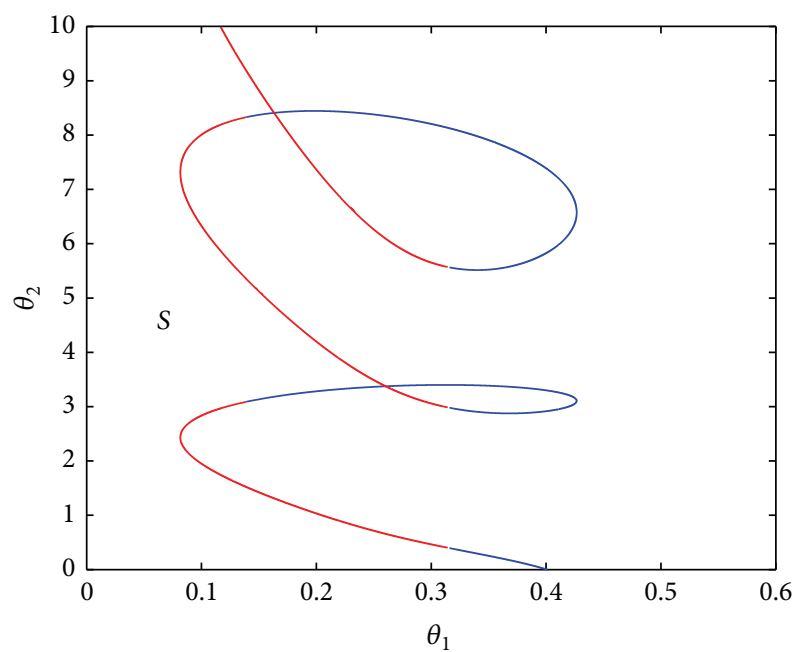

FIgURE 12: $\gamma=12$.

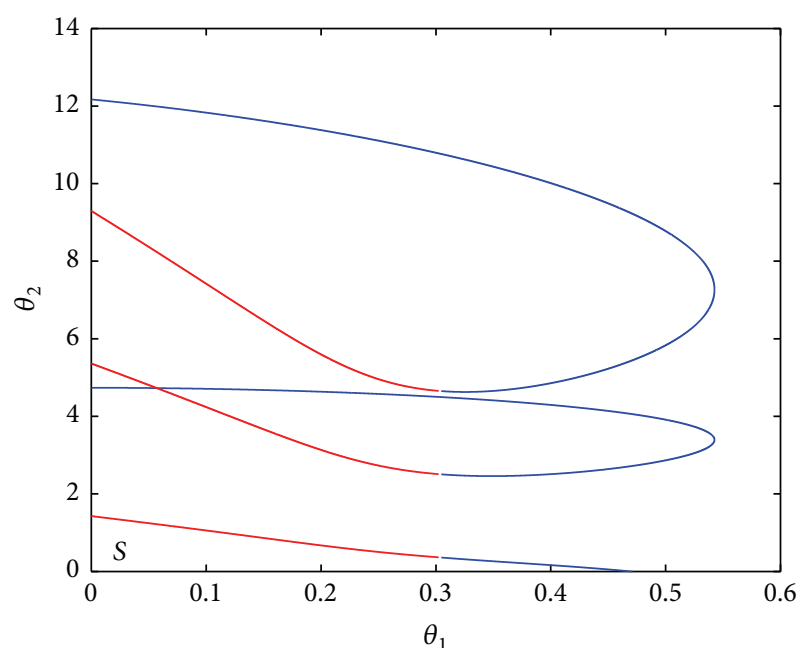

FIGURE 13: $\gamma=22$.

in $I_{Y}$ causes destabilization. However, due to the concavity of the crossing curve, there is a possibility that a decrease in $I_{K}$ causes destabilization. Thus, the existence of a lag complicates the effects of investment on stability.

Finally, we investigate the case wherein both fiscal and monetary policy lags exist. In this case, $I_{Y}$ and $I_{K}$ affect the stability condition (i.e., location of curve $M 0$ ) through $\omega \in \Omega$, $b_{1}$, and $b_{2}$. According to numerical simulations, increases in the absolute values of $I_{Y}$ and $I_{K}$ generally shift the curve $M 0$ toward the left. Therefore, these changes decrease the stability region. However, if $M 0$ completely shifts even to the negative region of $\theta_{1}$, then the curve that specifies the stability region is no longer $M 0$ but is $M 1$. For example, when $I_{Y}=3.0$, the stability region $S$ is defined as the left-hand side region of the curve $M 0$ in Figure 15. However, when $I_{Y}=4.0$, the stability region $S$ is defined as the left-hand side region of the curve $M 1$ in Figure 16. Thus, an increase in $I_{Y}$ may increase the stability region.

\section{Conclusion}

In this study, we analyzed the effects of fiscal and monetary policy lags on economic stability. In Section 3, we examined the case of a monetary policy with a lag. We confirmed that the results of Asada and Yoshida [12] that examined the case of a fiscal policy with a lag also basically hold in our model; that is, policy lags may cause economic instability and suppress the effect of expeditious policies aimed at economic stability (a model with a fiscal policy that does not consider a policy lag is proposed by Asada [21]).

Moreover, in Section 4, we examined the case wherein both fiscal and monetary policy lags exist and demonstrated that, in the neighborhood of a stability crossing curve, a monetary policy lag can have a stabilizing effect.

According to Friedman [22], policy lags can generally be classified into three types: recognition, implementation, and diffusion lags. Unlike recognition and diffusion lags, implementation lag can be considered as purposefully adjustable to 

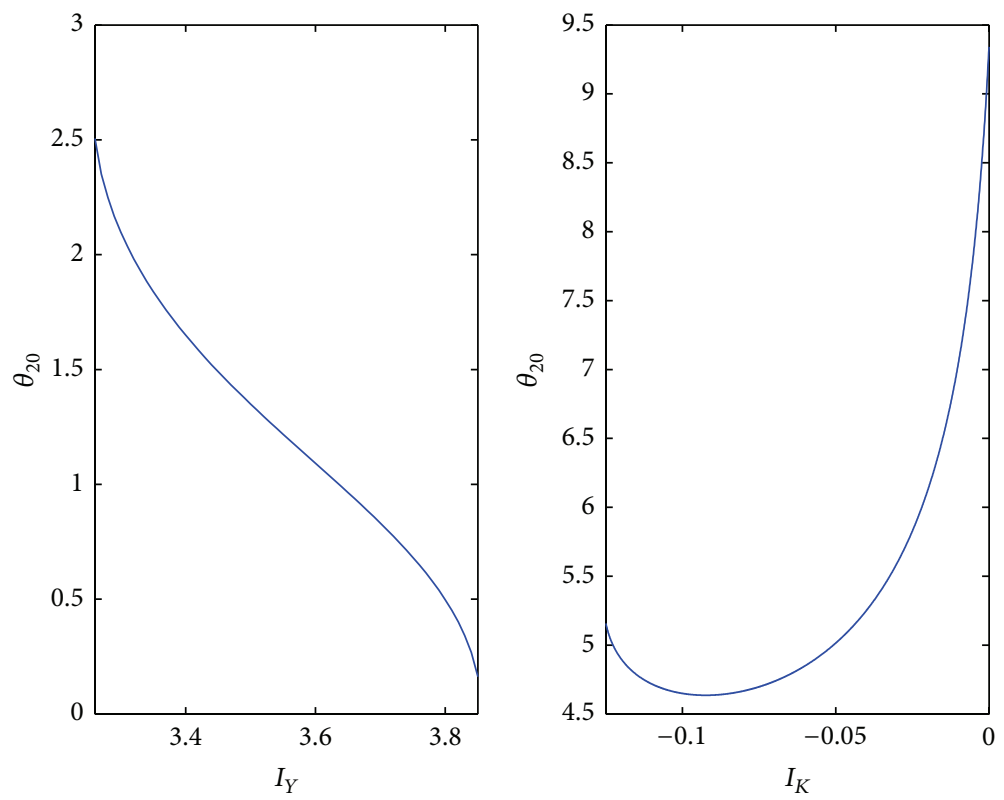

FIgURE 14: Crossing curve $\theta_{20}$.

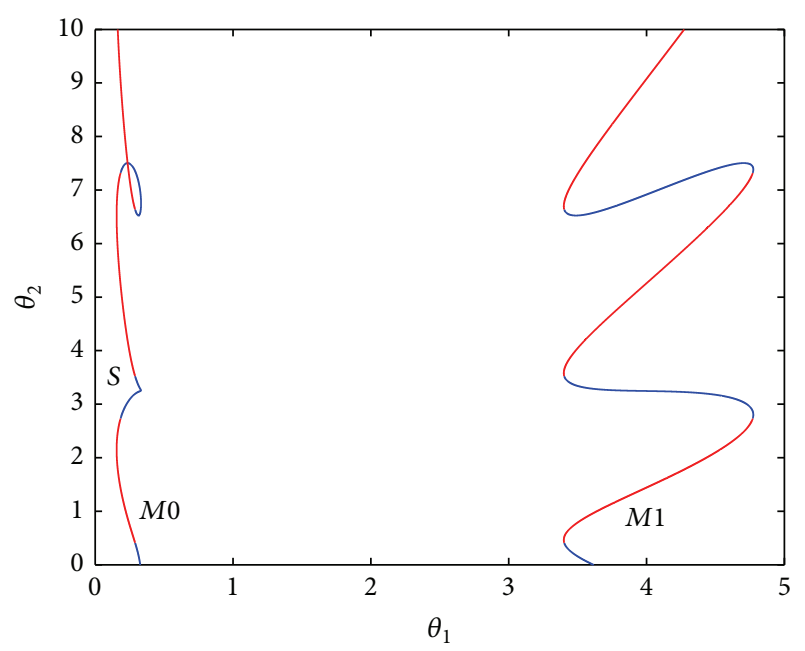

FIGURE 15: $I_{Y}=3.0$.

some extent. Therefore, this study suggests that an adjustment of the timing of policy implementation can be a means to achieve stabilization.

\section{Appendix}

\section{Direction of Crossing}

Differentiating (19) with respect to $\theta_{2}$, we obtain

$$
\begin{aligned}
& \left\{2 \lambda+\left(b_{1}+\alpha \beta\right)-\alpha A_{2} e^{-\theta_{2} \lambda}+\alpha A_{2} \lambda \theta_{2} e^{-\theta_{2} \lambda}\right\} \frac{d \lambda}{d \theta_{2}} \\
& =-\alpha A_{2} \lambda^{2} e^{-\theta_{2} \lambda}
\end{aligned}
$$

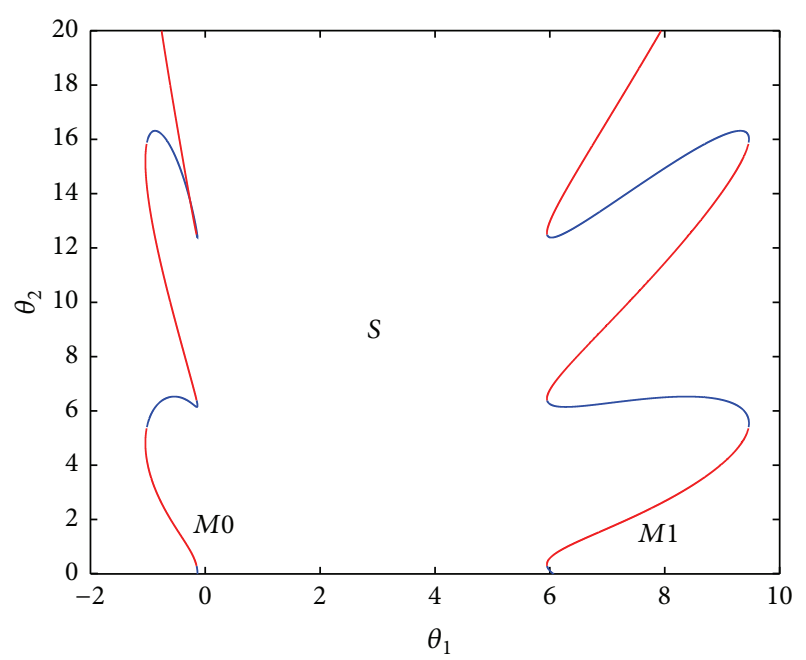

FIGURE 16: $I_{Y}=4.0$.

or, equivalently,

$$
\left(\frac{d \lambda}{d \theta_{2}}\right)^{-1}=\frac{-\left(2 \lambda+b_{1}+\alpha \beta\right) e^{\theta_{2} \lambda}}{\alpha A_{2} \lambda^{2}}+\frac{1}{\lambda^{2}}-\frac{\theta_{2}}{\lambda}
$$

From (19), we can express $e^{\theta_{2} \lambda}$ as follows:

$$
e^{\theta_{2} \lambda}=\frac{\alpha A_{2} \lambda}{\lambda^{2}+\left(b_{1}+\alpha \beta\right) \lambda+\left(b_{2}-\alpha \beta I_{K}\right)} .
$$


Substituting (A.3) into (A.2) and by evaluation at $\lambda=i \omega^{*}$, we obtain

$$
\begin{aligned}
\left.\operatorname{Re}\left(\frac{d \lambda}{d \theta_{2}}\right)^{-1}\right|_{\lambda=i \omega^{*}} & \operatorname{Re}\left[\frac{-\left(2 i \omega^{*}+b_{1}+\alpha \beta\right)}{i \omega^{*}\left\{-\omega^{* 2}+\left(b_{1}+\alpha \beta\right) i \omega^{*}+\left(b_{2}-\alpha \beta I_{K}\right)\right\}}-\frac{1}{\omega^{* 2}}\right. \\
- & \left.\frac{\theta_{2}}{i \omega^{*}}\right]=\frac{\left(b_{1}+\alpha \beta\right)^{2} \omega^{* 2}+2 \omega\left\{\omega^{* 3}-\left(b_{2}-\alpha \beta I_{K}\right) \omega^{*}\right\}}{\left\{\omega^{* 3}-\left(b_{2}-\alpha \beta I_{K}\right) \omega^{*}\right\}^{2}+\left\{\left(b_{1}+\alpha \beta\right) \omega^{* 2}\right\}^{2}} \\
- & \frac{1}{\omega^{* 2}} \\
= & \frac{2 \omega^{*}\left\{\omega^{* 3}-\left(b_{2}-\alpha \beta I_{K}\right) \omega^{*}\right\}-\left\{\omega^{* 2}-\left(b_{2}-\alpha \beta I_{K}\right)\right\}^{2}}{\left\{\omega^{* 3}-\left(b_{2}-\alpha \beta I_{K}\right) \omega^{*}\right\}^{2}+\left\{\left(b_{1}+\alpha \beta\right) \omega^{* 2}\right\}^{2}} .
\end{aligned}
$$

The denominator of the right-hand side of this expression is positive. Thus, we only have to observe the sign of the numerator. The numerator is expanded as $\omega^{* 4}-\left(b_{2}-\alpha \beta I_{K}\right)$. Furthermore, using (26), it is rewritten as follows:

$$
\omega^{* 4}-\left(b_{2}-\alpha \beta I_{K}\right)=\omega^{* 2} \sqrt{N} .
$$

As $N>0$, we obtain $\left.\operatorname{Re}\left(d \lambda / d \theta_{2}\right)^{-1}\right|_{\lambda=i \omega^{*}}>0$.

\section{Conflict of Interests}

The author declares that there is no conflict of interests regarding the publication of this paper.

\section{Acknowledgments}

The author appreciates the helpful comments and suggestions received from an anonymous referee and Professors Akio Matsumoto and Toichiro Asada (Chuo University).

\section{References}

[1] M. Kalecki, "A macrodynamic theory of business cycles," Econometrica, vol. 3, no. 3, pp. 327-344, 1935.

[2] M. Szydłowski, "Time-to-build in dynamics of economic models. I. Kalecki's model," Chaos, Solitons \& Fractals, vol. 14, no. 5, pp. 697-703, 2002.

[3] M. Szydłowski, "Time to build in dynamics of economic models. II. Models of economic growth," Chaos, Solitons \& Fractals, vol. 18, no. 2, pp. 355-364, 2003.

[4] L. Fanti and P. Manfredi, "Chaotic business cycles and fiscal policy: an IS-LM model with distributed tax collection lags," Chaos, Solitons \& Fractals, vol. 32, no. 2, pp. 736-744, 2007.

[5] H. Yoshida and T. Asada, "Dynamic analysis of policy lag in a Keynes-Goodwin model: stability, instability, cycles and chaos," Journal of Economic Behavior \& Organization, vol. 62, no. 3, pp. 441-469, 2007.

[6] G. J. Schinasi, "A nonlinear dynamic model of short run fluctuations," Review of Economic Studies, vol. 48, no. 4, pp. 649656, 1981.

[7] K. Sasakura, "On the dynamic behavior of Schinasi's business cycle model," Journal of Macroeconomics, vol. 16, no. 3, pp. 423444, 1994.
[8] L. De Cesare and M. Sportelli, "A dynamic IS-LM model with delayed taxation revenues," Chaos, Solitons \& Fractals, vol. 25, no. 1, pp. 233-244, 2005.

[9] A. Matsumoto and F. Szidarovszky, "Dynamics in delay ISLM model with tax collections," IERCU Discussion Paper 208, Chuo University, Hachioji, Japan, 2013.

[10] N. Kaldor, "A model of the trade cycle," The Economic Journal, vol. 50, no. 197, pp. 78-82, 1940.

[11] W. W. Chang and D. J. Smyth, "The existence and persistence of cycles in a non-linear model: Kaldor's 1940 model re-examined," The Review of Economic Studies, vol. 38, no. 1, pp. 37-44, 1971.

[12] T. Asada and H. Yoshida, "Stability, instability and complex behavior in macrodynamic models with policy lag," Discrete Dynamics in Nature and Society, vol. 5, no. 4, pp. 281-295, 2001.

[13] L. Zhou and Y. Li, "A dynamic IS-LM business cycle model with two time delays in capital accumulation equation," Journal of Computational and Applied Mathematics, vol. 228, no. 1, pp. 182187, 2009.

[14] J. Cai, "Hopf bifurcation in the IS-LM business cycle model with time delay," Electronic Journal of Differential Equations, vol. 15, pp. 1-6, 2005.

[15] M. Sportelli, L. De Cesare, and M. T. Binetti, "A dynamic ISLM model with two time delays in the public sector," Applied Mathematics and Computation, vol. 243, pp. 728-739, 2014.

[16] A. Matsumoto and F. Szidarovszky, "Nonlinear multiplieraccelerator model with investment and consumption delays," Structural Change and Economic Dynamics, vol. 33, pp. 1-9, 2015.

[17] K. Gu, S.-I. Niculescu, and J. Chen, "On stability crossing curves for general systems with two delays," Journal of Mathematical Analysis and Applications, vol. 311, no. 1, pp. 231-253, 2005.

[18] G. Gandolfo, Economic Dynamics, Springer, Berlin, Germany, 4th edition, 2010.

[19] A. Matsumoto and F. Szidarovszky, "An elementary study of a class of dynamic systems with two time delays," Cubo, vol. 14, no. 3, pp. 103-113, 2012.

[20] R. E. Bellman and K. L. Cooke, Differential-Difference Equations, Academic Press, New York, NY, USA, 1963.

[21] T. Asada, "Government finance and wealth effect in a Kaldorian cycle model," Journal of Economics, vol. 47, no. 2, pp. 143-166, 1987.

[22] M. Friedman, "A monetary and fiscal framework for economic stability," The American Economic Review, vol. 38, no. 3, pp. 245264, 1948. 


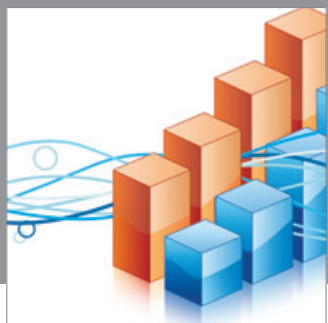

Advances in

Operations Research

mansans

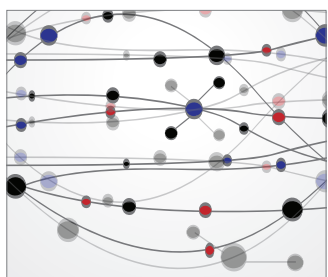

The Scientific World Journal
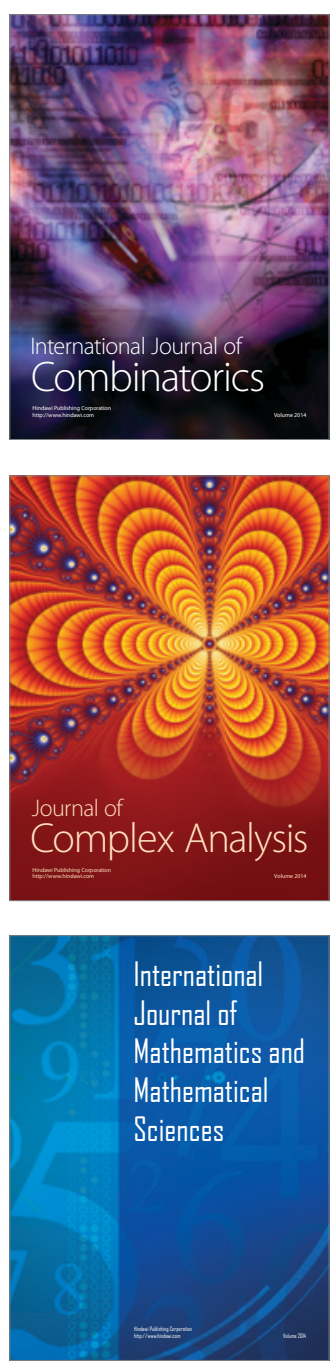
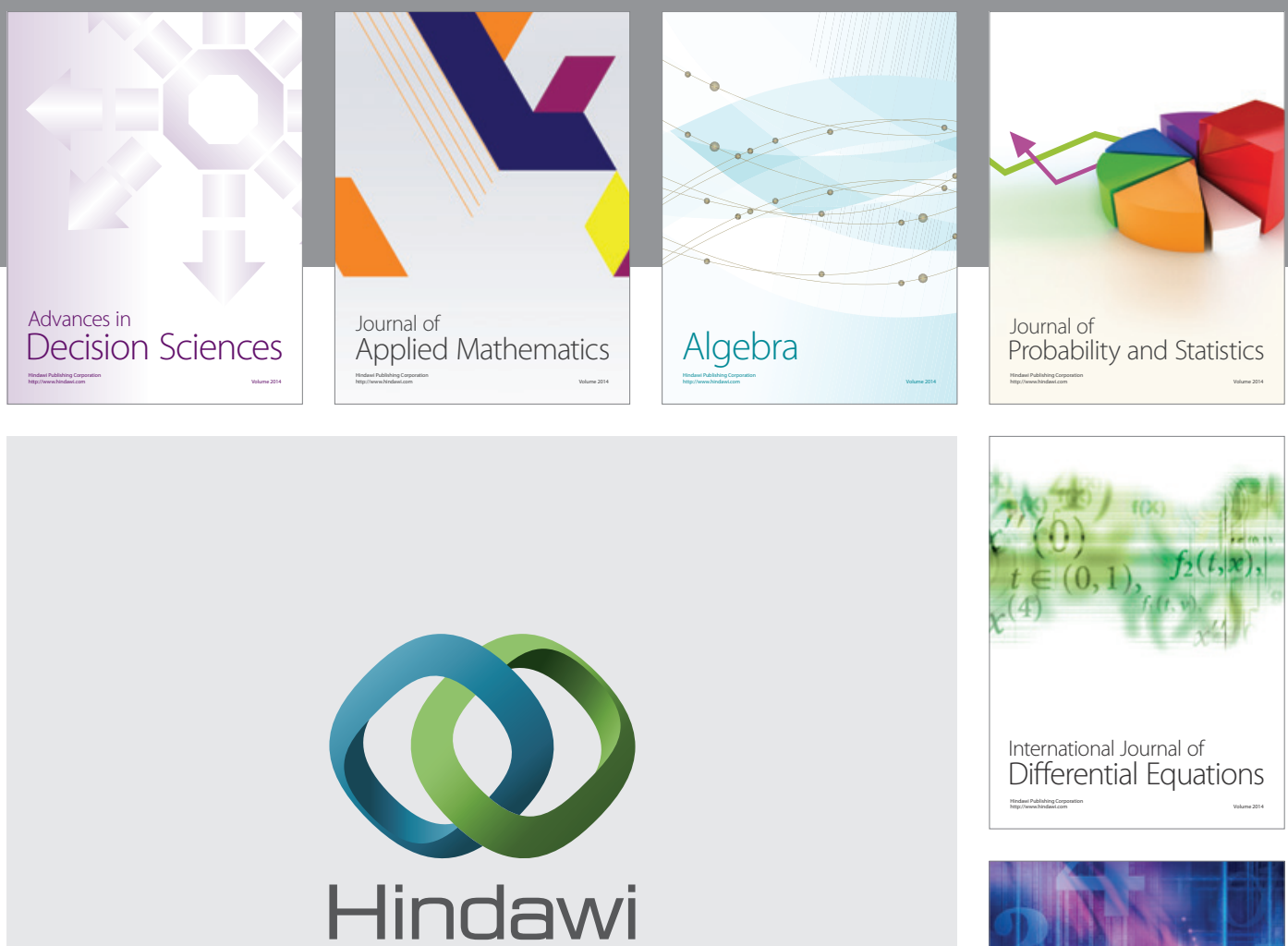

Submit your manuscripts at http://www.hindawi.com
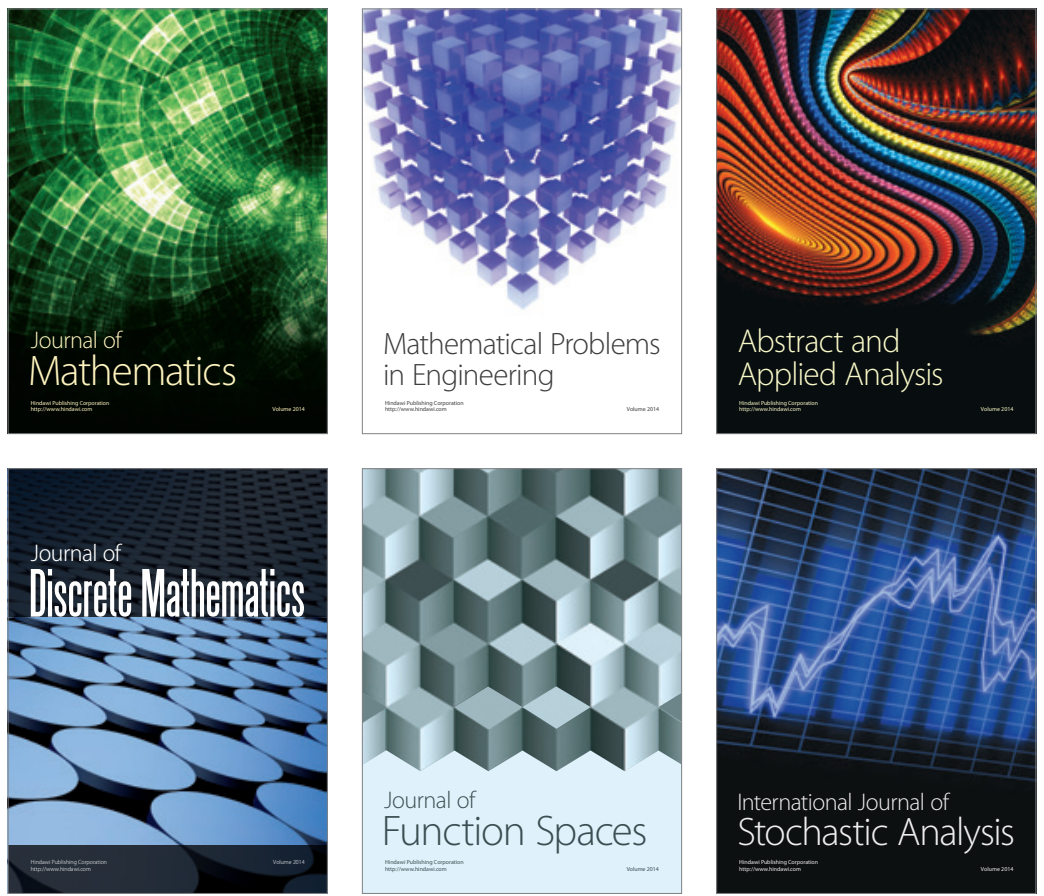

Journal of

Function Spaces

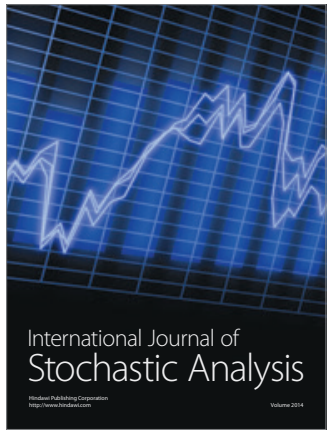

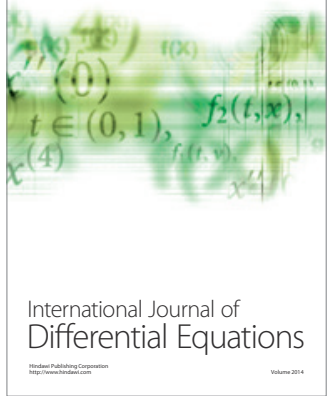
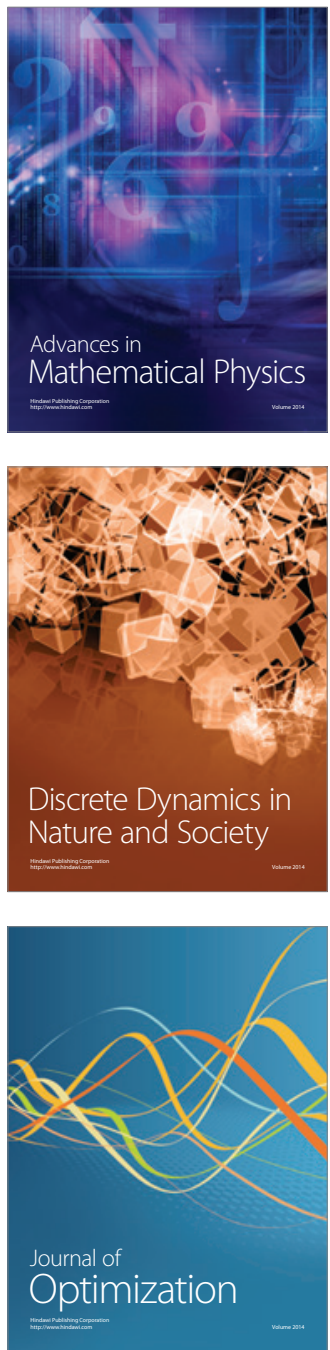